\title{
Algunas aplicaciones de la nanofotónica en la biomedicina $^{\diamond}$
}

\section{Some applications of nanophotonics in biomedicine}

\author{
Elder De la Rosa,,,• Gonzalo Ramírez,** Sandeep Panikar,,*** \\ Tanya Camacho," Pedro Salas, "† Tzarara López-Luke"*t
}

\begin{abstract}
In this work, the optical and electronic properties of nanomaterials are discussed, its fundamental characteristics and its application in the design of devices and techniques for detection, imaging and therapy, especially in cancer problems, are analyzed. Some recent results obtained in our laboratory where we have been able to measure concentrations of the order of $10^{-22}$ moles of complexes of interest are discussed. We report the detection of residues in blood of medications of the order of $10^{-8}$ and $10^{-9}$ molar $(\mathrm{M})$, which opens the way for drug monitoring with a focus on the implementation of a personalized medicine. We discuss results of therapy techniques with the use of nanomaterials that have allowed us to reduce cell viability below $10 \%$. These results show that nanotechnology is changing the health paradigm to preventive medicine, personalized and available to everyone.
\end{abstract}

KEYWORDS: nanotechnology, nanoparticles, SERS, nanophotonics, bionanophotonics, nanomedicine, teranotic medicine.

RESUMEN: En este trabajo se discuten las propiedades ópticas y electrónicas de nanomateriales, se analizan sus características fundamentales y su aplicación en el diseño de dispositivos y técnicas para la detección, imagen y terapia, especialmente en problemas de cáncer. Se discuten algunos resultados recientes obtenidos en nuestro laboratorio donde hemos podido medir concentraciones del orden de $10^{-22}$ moles de complejos de interés. Reportamos la detección de residuos en sangre de medicamentos del orden de $10^{-8}$ y $10^{-9}$ molar (M), lo que abre el camino para el monitoreo de fármacos con un enfoque a la implementación de una medicina persona-

Recibido: 2 de septiembre de 2019 .

Aceptado: 18 de octubre de 2019.

- Agradecemos al Conacyt por el apoyo financiero para el desarrollo de esta investigación a través del proyecto con referencia 259192, y a la Universidad De La Salle Bajío por el apoyo a través del proyecto 19-021. Sandeep Panikar agradece, a Conacyt por su beca doctoral.

* Facultad de Ingenierías, Universidad De La Salle Bajio, Campus Campestre, León, Guanajuato, 37150, México.

** Centro de Investigación en Química Aplicada, COITTEC. 140, Blvd. Enrique Reyna, Saltillo, 25294, México.

*** Facultad de Ingenierías, Universidad De La Salle Bajio, Campus Campestre. Unidad de Biotecnología Médica y Farmacéutica, Centro de Investigación y Asistencia en Tecnología y Diseño del Estado de Jalisco, México.

- Unidad de Biotecnología Médica y Farmacéutica, Centro de Investigación y Asistencia en Tecnología y Diseño del Estado de Jalisco.

- Centro de Física Aplicada y Tecnología Avanzada, Universidad Nacional Autonoma de México, AP 1-1010 Querétaro, Qro. 76000 México.

"* Instituto de Investigación en Metalurgia y Materiales, Universidad Michoacana de San Nicolás de Hidalgo, Ciudad Universitaria, Morelia, 58030, México.

- Autor de correspondencia: edelarosa@delasalle.edu.mx 
lizada. Discutimos resultados de técnicas de terapia con el uso de nanomateriales que han permitido reducir la viabilidad celular por debajo del $10 \%$. Estos resultados muestran que la nanotecnología está cambiando el paradigma en salud a una medicina preventiva, personalizada y al alcance de todos.

PALABRAS CLAVE: nanotecnología, nanopartículas, SERS, nanofotónica, bionanofotónica, nanomedicina, medicina teranóstica.

\section{Introducción}

EN ESCALA nanométrica, las propiedades de los materiales, o más precisamente nanomateriales, están determinadas por el tamaño y forma de la partícula donde el comportamiento de los electrones esta definido por efectos cuánticos <https://www.nano.gov/timeline>. Para tener una idea del tamaño, considere que un nanómetro ( $\mathrm{nm}$ ) es una mil millonésima parte de un metro, o bien que en un milímetro caben un millón de nanómetros, o bien considere que el ancho de una doble cadena de ADN mide entre 2.2 y 2.6 nanómetros. Es decir, la nanoescala es donde ocurren muchos de los fenómenos biológicos y en este sentido los nanomateriales tienen una gran oportunidad de aplicación en el área biomédica.

Por sus propiedades, estos nanomateriales encuentran aplicaciones diversas prácticamente en todas las áreas y aportan soluciones a problemas fundamentales que aquejan a la humanidad (Lauterwasser, 2005; US National Science and Technology Council, 1999; Burda et al., 2005; Roew et al., 1999; Pitkethly, 2004). Con los nanomateriales se diseñan textiles inteligentes, materiales más resistentes y más ligeros para la construcción y transporte (Cherenack y Van Pieterson, 2012; Das y Mitra, 2014). Se impacta en la industria electrónica y de comunicaciones en el diseño de nuevos dispositivos que están definiendo las nuevas tecnologías de comunicación (Yin y Talapin, 2013; Choi et al., 2016; Senellart et al., 2017).

Se diseñan nanomateriales para proteger el medio ambiente, pues con este tipo de materiales se planean celdas solares de última generación, se implementan procesos para la limpieza de agua y se proyectan sensores para la detección de contaminantes (Kumar et al., 2017; Savage y Diallo, 2005; Khin, 2012). En medicina y salud se diseñan biosensores para detección temprana de compuestos asociados con problemas de salud, se usan nanomateriales para obtener imágenes de áreas dañadas que permiten una identificación rápida y precisa, y se implementan diferentes tipos de terapia, entre ellas terapia fototérmica, terapia fotodinámica y la entrega dirigida de medicamentos (Riehemann et al., 2009; Shi et al., 2017; Lammers et al., 2011; Bai et al., 2015). Desde su nacimiento, la nanotecnología se ha considerado como la tecnología del futuro, siendo esta cada vez más presente.

Las propiedades ópticas y electrónicas de los nanomateriales dan origen a la nanofotónica: ciencia y tecnología para la generación, absorción, manipulación, reflexión, esparcimiento y detección de fotones. La interacción de estos nanomateriales o sistemas nanoestructurados con la materia biológica 
da lugar a la bionanofotónica y, más en particular, a la nanomedicina (Riehemann et al., 2009; Shi et al., 2017). En esta última, y gracias a la nanotecnología, se ha desarrollado la medicina teranóstica, aproximación que contempla el uso de nanocomplejos capaces de funcionar para la detección, imagen, terapia y recabar información sobre el efecto de la terapia de manera simultánea. Es un coctel que identifica el problema de salud, introduce la terapia y coadyuva a determinar el efecto de la misma (Lammers et al., 2011; Bai et al., 2015).

En este trabajo, presentamos una descripción de las propiedades de diferentes tipos de nanomateriales que tienen potencial para ser utilizados en aplicaciones bionanofotónicas y biomédicas. Posteriormente, presentamos resultados recientes obtenidos en nuestro grupo, sobre el uso de dichos nanomateriales relevantes en el diseño de biosensores, detección, imagen y diferentes tipos de terapia contra cáncer, incluyendo el uso de complejos teranósticos que combinan los diferentes tipos de terapia.

\section{Fundamentos}

\section{Puntos cuánticos y el efecto de confinamiento}

Las nanopartículas (NPs) más utilizadas en bionanofotónica para aplicaciones biomédicas o nanomedicina son los puntos cuánticos, las nanopartículas cerámicas y fluoradas, y las nanopartículas metálicas. Los puntos cuánticos (QDs, por sus siglas en inglés) son NPs semiconductoras que presentan el llamado efecto de confinamiento cuántico. Este fenómeno ocurre cuando el movimiento de los electrones queda confinado en al menos una dirección menor a $100 \mathrm{~nm}$. El confinamiento puede ser débil, mediano y fuerte para una, dos y tres dimensiones, respectivamente. El resultado del confinamiento es una descomposición de las bandas de energía a niveles discretos de energía y un ensanchamiento de la banda prohibida. La máxima intensidad de confinamiento y, con ello, el máximo ensanchamiento de la banda prohibida, ocurre cuando el radio de Bohr del excitón $\mathrm{R}_{\mathrm{Bexc}}$ (separación del par electrón-hueco (e-h)), que se forma cuando se excita un electrón de la banda de valencia a la banda de conducción del semiconductor, tiene un tamaño comparable al diámetro de la NP. Como es de esperarse, depende de la composición del material, obteniéndose un valor típico entre 3 y $5 \mathrm{~nm}$ para el caso de CdSe. Cuanto más comparable sea el radio de Borh y el diámetro de la nanopartícula, mayor será el ensanchamiento. El resultado neto de este fenómeno es la capacidad de sintonizar la emisión de un QD controlando su tamaño (figura 1), (Bera et al., 2010; Pu et al., 2018). Los QDs son altamente brillantes, sin embargo presentan defectos de superficie que promueven relajaciones no radiativas reduciendo su eficiencia de emisión; esto se resuelve agregando una capa sobre la superficie de un material con una banda prohibida mayor formando una nanopartícula tipo núcleo/coraza con la que se han reportado eficiencias cuánticas del 90\% (Pu et al., 2018; Yang et al., 2015). 
FIGURA 1. a) Diagrama esquemático que muestra la discretización de las bandas de energía y el ensanchamiento de la banda prohibida para un punto cuántico, $\lambda_{1}$ se refiere al fotón que excita el electrón de la banda de valencia a la banda de conducción y $\lambda_{2}$ se refiere al fotón (fluorescencia) que resulta de la recombinación del par electrón-hueco; b) diagrama esquemático que muestra el radio de Bohr del excitón $\left(R_{\text {Bexc }}\right)$ y un punto cuántico de radio $r$; $c$ ) fotografía de puntos cuánticos de CdSe con diámetros entre 3 y $6 \mathrm{~nm}$, obtenidos en nuestro laboratorio por el método de inyección caliente ampliamente reportado en la literatura, ver, por ejemplo (Bera et al., 2010; Pu et al., 2018; Yang et al., 2015).

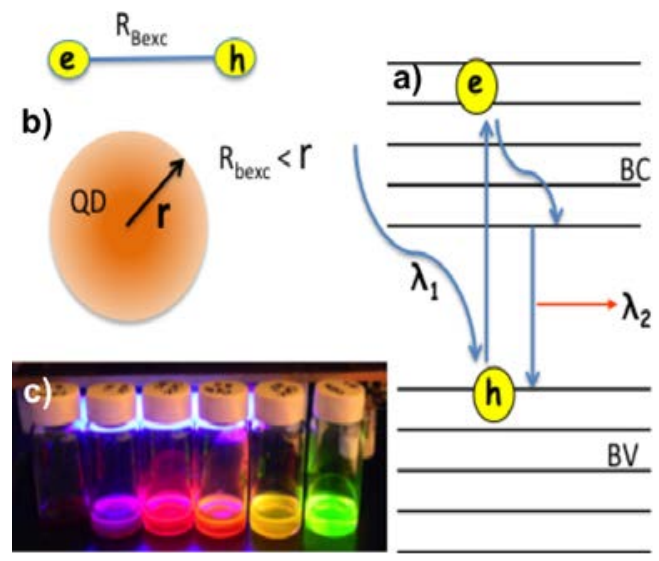

Fuente: Elaboración de los autores.

\section{Nanopartículas dopadas con lantánidos $\left(\operatorname{Ln}^{3+}\right)$}

Otra forma de obtener una señal luminosa altamente sintonizable es dopando con lantánidos $\left(\mathrm{Ln}^{3+}\right)$ a las NPs de óxidos, oxisulfuros, oxifloruros o fluoruros. En este caso, el color o longitud de onda de la señal emitida se obtiene del lantánido que se elija. No hay confinamiento cuántico pues los lantánidos ya tienen niveles discretos de energía y el compuesto que lo soporta requiere tamaños menores a $1 \mathrm{~nm}$ para presentarlo. Sin embargo, el tamaño de la NP y su alta área superficial pueden modificar la interacción entre los lantánidos y la energía fonónica o vibración de la red cristalina del soporte. Esto da como resultado relajaciones del ion dopante que en otras condiciones no ocurren, dando lugar a emisiones que típicamente no suceden o lo hacen en forma más eficiente. Hay dos maneras de producir luz con los lantánidos. Una es cuando se excita con una longitud de onda $\lambda_{1}$ y se obtiene una señal de menor energía con longitud de onda $\lambda_{2}$. En este caso se habla de conversión descendente $o$ simplemente fotoluminiscencia. Los iones más utilizados son $\mathrm{Eu}^{3+}$ (rojo), $\mathrm{Tb}^{3+}$ (verde), $\mathrm{Sm}^{3+}$ (naranja), Ce ${ }^{3+}$ (amarillo) (Yuan, Wang, 2006; De la Rosa-Cruz et al., 2003). Todos son utilizados para producir luz de diferentes colores, pero el último es el que hace posible la obtención de LEDs de luz blanca (figura 2).

El otro caso es cuando se excita con una longitud de onda $\lambda_{1}$ de menor energía que la señal obtenida $\lambda_{2}$. En este caso, se excita con luz en el cercano infrarrojo (NIR) y se obtiene luz visible. A este fenómeno se le conoce como conversión ascendente (UC, por sus siglas en inglés); es un fenómeno cooperativo pues requiere la absorción de al menos dos fotones de baja energía 
Mundo Nano | ARTículos DE INVESTIGACIÓN | www.mundonano.unam.mx 13(24), 1e-24e, enero-junio 2020 | http://dx.doi.org/10.22201/ceiich.24485691e.2020.24.69618 Elder De la Rosa, Gonzalo Ramírez, Sandeep Panikar, Tanya Camacho, Pedro Salas, Tzarara López-Luke

para producir un fotón de mayor energía. En la mayoría de los casos se utiliza $\mathrm{Yb}^{3+}$ como absorbedor de la luz de excitación por su alta sección transversal de absorción y transfiere su energía a otros iones que tienen niveles de energía resonantes con el único nivel que presenta el $\mathrm{Yb}^{3+}$. Con la combinación adecuada de diferentes iones es posible obtener emisión en todo el rango visible del espectro incluyendo luz blanca. Los iones más estudiados son el $\mathrm{Er}^{3+}$ que produce bandas de emisión dominantes en el verde y el rojo; el $\mathrm{Tm}^{3+}$ con bandas de emisión en el azul y rojo; el $\mathrm{Ho}^{3+}$ con bandas en el verde y rojo; el $\mathrm{Pr}^{3+}$ con emisión roja (figura 2), (Gnach, Bednarkiewicz, 2012; Wang, Liu, 2014; Solis et al., 2010).

\section{Nanopartículas metálicas}

Las nanopartículas metálicas son, sin duda, las más atractivas para aplicaciones en bionanofotónica, especialmente las NPs de oro. En este caso, los electrones de superficie de la NP forman un plasma que oscila con la frecuencia del campo eléctrico incidente. Esta oscilación coherente de los electrones en la banda de conducción a una frecuencia de resonancia es a lo que se le llama plasmón de superficie localizada resonante (LSPR, por sus siglas en inglés) y depende de la densidad de electrones $f$, del medio efectivo, del índice de re-

FIGURA 2. a) Se muestran las bandas de emisión verde y roja del $\mathrm{Er}^{3+}$ después de ser excitado a 980 $\mathrm{nm}$, a diferentes concentraciones de $\mathrm{Yb}^{3+} ;$ b) fotografía de los diferentes colores que es posible obtener mediante la combinación adecuada de $\mathrm{Ln}^{3+}$; la última línea de colores es el resultado de variar la concentración de $\mathrm{Yb}^{3+}$ a una concentración fija de $\mathrm{Er}^{3+}$, la penúltima corresponde a una variación de la concentración de $\mathrm{Er}^{3+}$ a una concentración fija de $2 \mathrm{~mol} \%$ de $\mathrm{Yb}^{3+}$, mientras que la antepenúltima línea, de izquierda a derecha, se obtiene utilizando $\mathrm{Tm}^{3+}$ (azul y rosa), $\mathrm{Er}^{3+}$ (verde y rojo) y la luz blanca se obtiene combinando $\mathrm{Yb}^{3+}, \mathrm{Er}^{3+}, \mathrm{Tm}^{3+} ; \mathrm{c}$ ) diagrama de energía característico del $\mathrm{Yb}-\mathrm{Er}$.
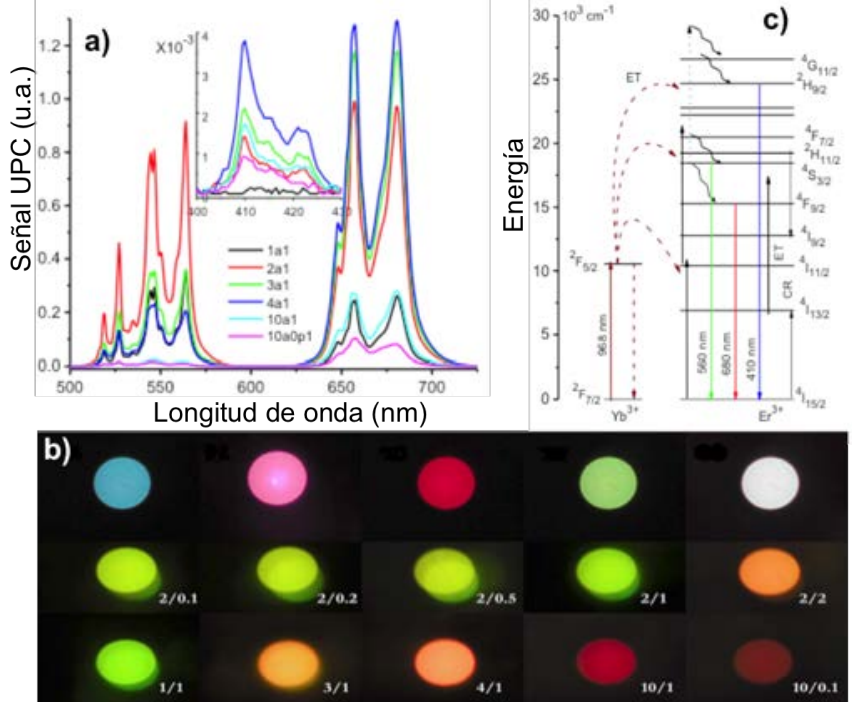

Fuente: Tomado de: Solis et al. (2010). 
fracción $n$, del tamaño $r$, de la forma de la NP y de la separación entre ellas. Es decir, la posición espectral del LSPR se sintoniza cambiando el tamaño, la forma de la nanopartícula metálica y las propiedades del medio donde se encuentra (figura 3). El coeficiente de extinción del plasmón de resonancia está compuesto por el coeficiente de absorción y el de esparcimiento, $C_{\text {ext }}=C_{a b s}+$ $C_{e s p}$, es decir, parte de los fotones incidentes son absorbidos y parte son esparcidos mayoritariamente en forma elástica (C. Noguez, 2007). Cuando la $\mathrm{NP}$ es pequeña, $C_{e x t}$ está dominado por el coeficiente de absorción $C_{a b s}$. En este caso, la luz absorbida induce un incremento de temperatura entre 5 y 10 ${ }^{\circ} \mathrm{C}$ suficiente para inducir la muerte celular, por lo cual es un excelente candidato para usarse en terapia fototérmica. Sin embargo, para nanopartículas esféricas pequeñas $(<70 \mathrm{~nm}$ ) la posición del plasmón de resonancia está localizada en la parte visible del espectro que tiene muy baja penetración en el tejido. Cuando se incrementa el tamaño de la NP o cambia su forma, el coeficiente de extinción está dominado por el esparcimiento de la luz y el plasmón de resonancia se corre al cercano infrarrojo. La luz en el cercano infrarrojo tiene mejor penetración en el tejido, pero al presentar baja absorción la terapia fototérmica no es viable. Para superar este problema, se han desarrollado otras aproximaciones para obtener altos niveles de absorción en la parte del cercano infrarrojo del espectro (Pissuwan et al., 2006; Huang et al., 2006; Gobin et al., 2007). Además, cuando se tienen NP en forma de estrellas, estas pueden depolarizar la luz incidente mientras que las esferas la mantienen. Este fenómeno, además del esparcimiento, es ampliamente utilizado para el diseño de biosensores. La sección transversal de absorción es $\sigma_{a b s} \alpha f$ y la sección transversal del esparcimiento es $\sigma_{e s p} \alpha f^{2}$ para una partícula en general. Para una NP metálica $f \approx 10^{-5}$ mientras que para una molécula $f \approx 1$. Es decir, $\sigma_{a b s}$ and $\sigma_{e s p}$ es cinco y diez órdenes de magnitud mayor que una molécula, respectivamente. Esto se explica porque las NPs metálicas son

FIGURA 3. Gráfico que muestra la sintonización de la posición del plasmón de resonancia localizado de una NP metálica como resultado del cambio de forma de la NP, en este caso corresponde a una nanopartícula de plata.

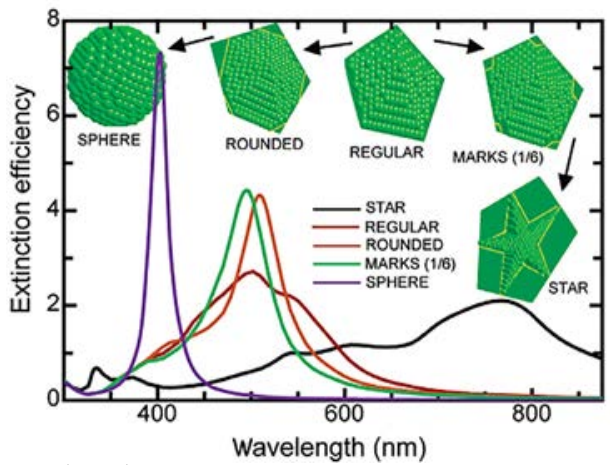

Fuente: Tomado de Noguez (2007).

Wavelength $(\mathrm{nm})$ 
mejores absorbedores y mucho mejores produciendo esparcimiento, lo cual las hace ideales para el diseño de biosensores (Bauch et al., 2014; Ament et al., 2012; Oliverio et al., 2017).

Cuando la luz incide sobre una molécula, la mayoría de los fotones se esparcen elásticamente, esto se conoce como esparcimiento Rayleigh. Pero hay una pequeña fracción de fotones, aproximadamente 1 de cada $10^{6}$ fotones, que se esparcen en forma inelástica, esto es, sufren dispersión óptica a diferentes frecuencias de los fotones incidentes. A esta señal se le conoce como dispersión Raman y da lugar a la espectroscopía Raman. La señal, aunque muy pequeña, es una firma de la molécula que la produce y está definida por los enlaces que la forman, su estructura, esfuerzos sobre la muestra y el medio donde se encuentra. Estas características hacen de la espectroscopía Raman una técnica muy poderosa para la identificación de compuestos y el diseño de biosensores, siempre y cuando se pueda amplificar la señal.

La señal Raman se amplifica cuando la molécula de interés se coloca lo suficientemente cerca de una NP metálica, para que experimente la amplificación del campo eléctrico producida por la oscilación del plasma de electrones. El factor de calidad Q es una medida del número de oscilaciones y del incremento de la amplitud del campo local y se calcula como la razón entre la parte real e imaginaria del campo eléctrico: $Q \alpha-\operatorname{Re} E_{m} / \operatorname{Im} E_{m} 10<Q<100$. El factor de realce del campo local $E F \quad \alpha Q^{2} \rightarrow 10^{2}-10^{4}$ que corresponde al factor de realce de la absorción y emisión de una molécula adsorbida sobre la superficie de una NP metálica. La señal Raman amplificada por superficie (SERS, por sus siglas en inglés) es un proceso de dos fotones por lo que el factor de realce experimentado es $E F \propto Q^{4} \rightarrow 10^{4}-10^{8}$; no obstante, cuando se considera un sistema en resonancia y la forma de la NP como estructuras que amplifican mayormente el campo eléctrico como el efecto nanoantena, es posible alcanzar factores de realce de $10^{14}$ (Chorsi et al., 2017; Sharma et al., 2012; Kneipp et al., 2008; Li et al., 2015).

\section{Bionanosensores}

Los bionanosensores pueden ser clasificados de acuerdo con el tipo de mecanismo de transducción usado para la generación de señales como, electroquímicos, piezoeléctricos y ópticos. Estos últimos poseen la capacidad de detectar variaciones en las propiedades relacionadas con la radiación electromagnética como la longitud de onda, el tiempo de decaimiento, la intensidad de emisión o luz esparcida tanto elástica como inelástica (Raman) y la polarización de la luz (Kneipp et al., 2008; Li et al., 2015; Shao et al., 2010; Sun et al., 2019; Srivastava et al., 2017). Son de bajo costo de fabricación, de alta portabilidad, gran sensibilidad y selectividad, tiempo de respuesta rápida y gran resolución espacial. En los últimos años, se han desarrollado diversos nanosensores basados en luminiscencia utilizando materiales fluorescentes como colorantes orgánicos, QDs y nanopartículas metálicas (Oliverio et al., 2017; Chorsi et al., 
2017; Sharma et al., 2012; Kneipp et al., 2008; Li et al., 2015; Shao et al., 2010; Sun et al., 2019; Srivastava et al., 2017; Hildebrand, 2011).

Sin embargo, estos materiales requieren ser excitados con luz ultravioleta-visible, lo cual introduce ruido de fondo derivado de los procesos de autofluorescencia. Fenómeno que podría llegar a limitar su adecuado funcionamiento, así, actualmente se buscan nuevos materiales luminiscentes capaces de evitar esas limitaciones.

\section{Fluorescencia}

Las nanopartículas con conversión ascendente (UCNPs), como discutimos antes, convierten la luz de excitación en el cercano infrarrojo (NIR) en emisiones en las regiones ultravioleta, visible, o cercano infrarrojo de menores longitudes de onda mediante un proceso de absorción de múltiples fotones. Dichos materiales presentan varias ventajas para el desarrollo de nanosensores, la detección de biomarcadores, el escaneo óptico y algunas otras aplicaciones respecto a los fluoróforos mencionados anteriormente. Con el uso de UCNPs se elimina la autoflorescencia (con ello se mejora la relación señal/ ruido) y se mejora la resistencia a la fotodegradación. Presentan, asimnismo, emisiones intensas, sintonizables y modulables, baja toxicidad, prolongados tiempos de vida media de emisión, así como eliminación de los efectos causados por la luz de alta energía necesaria para la excitación (De la Rosa-Cruz et al., 2003; Gnach y Bednarkiewicz 2012; Wang y Liu, 2014; Solis et al., 2010; Dong et al., 2015).

Existen diferentes métodos para la síntesis de NPs, entre ellas, sol-gel, precipitación, combustión, hidrotermal, entre otras. Cualquiera que sea el método utilizado, es necesario optimizarlo para obtener NPs con tamaño controlado, estructura cristalina y morfologías específicas (figura 4). Gracias a ello, las UCNPs pueden ser usadas como donadoras en sistemas de detección basados en transferencia de energía por resonancia de fluorescencia (FRET). Este fenómeno es un proceso no radiativo que ocurre entre un donador y un aceptor de energía, y para que ocurra existen dos condiciones primordiales: i) las moléculas del donador y aceptor deben estar muy próximas entre sí (10-100 ̊̊), y, ii) el espectro de absorción del aceptor se debe traslapar con el espectro de emisión de fluorescencia del donador. De manera general, los mecanismos de detección en los nanosensores basados en transferencia de energía por resonancia pueden ser activados modulando la superposición de los espectros de emisión/excitación, o variando la distancia entre el emisor y el receptor. Para manipular la eficiencia del proceso FRET, la absorción de los aceptores debe mostrar cambios significativos (en intensidad o longitud de onda) después de reaccionar con el analito, ya sea para acoplarse con la emisión del donador o para desacoplarse. De ese modo, la emisión será, respectivamente, apagada o recuperada de manera gradual en función de la concentración del analito, con lo que se puede llevar a cabo su cuantificación (Hildebrand, 2011; Dong et al., 2015; Dvide Giust et al., 2018; Gu, Zhang 2018). 
Mundo Nano | ARTículos DE INVESTIGACIÓN | www.mundonano.unam.mx 13(24), 1e-24e, enero-junio 2020 | http://dx.doi.org/10.22201/ceiich.24485691e.2020.24.69618 Elder De la Rosa, Gonzalo Ramírez, Sandeep Panikar, Tanya Camacho, Pedro Salas, Tzarara López-Luke

FIGURA 4. Micrografias obtenidas mediante STEM de nanopartículas de $\mathrm{NaYF}_{4}$ : Yb,Er y NaYF $4: \mathrm{Yb}, \mathrm{Tm}$ preparadas por el método hidrotermal.

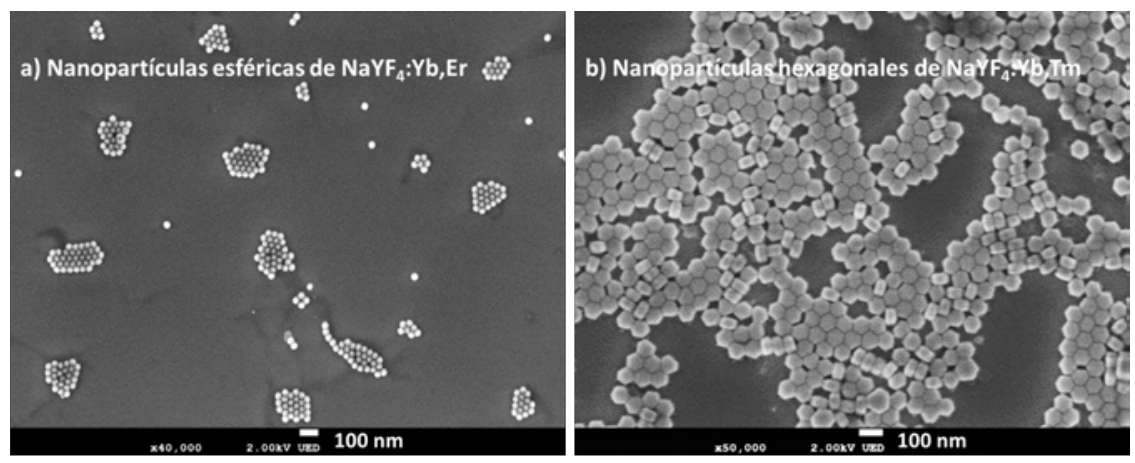

Fuente: Información detallada para la síntesis de este tipo de NPs puede encontrarse en: G. RamírezGarcía et al. (2018 y 2019).

Como plataforma de análisis de biomarcadores de enfermedades, los nanomateriales también han sido utilizados principalmente para aumentar la sensibilidad y especificidad analítica al ser acoplados a moléculas de interés biológico o farmacéutico. Un ejemplo de estas aplicaciones es el desarrollo de tiras de flujo laminar para la detección cualitativa y cuantitativa de microorganismos patógenos (figura 5). En este esquema, la solución que contiene el complejo de interés se deposita en la almohadilla de muestra y fluye por difusión hacia la almohadilla donde se deposita el nanoconjugado. El nanoconjugado, que consiste de NPs unidas a anticuerpos específicos que se adhieren o atrapan al complejo de interés, fluye hacia la línea de prueba. La línea de prueba contiene anclados los mismos anticuerpos que contienen los nanoconjugados de manera que atrapa el complejo de interés que está atrapado a la NP formando una estructura tipo sandwich. Si el complejo de interés no está presente, el nanoconjugado no es atrapado y sigue su camino hacia la línea de control. La línea de control contiene complejos que atrapan al anticuerpo del nanoconjugado fijándolo en ese espacio. Los nanoconjugados atrapados, ya sea en la línea de prueba o de control, producen una señal luminosa observable a simple vista cuando se excita con la longitud de onda apropiada, indicando la presencia o no del complejo de interés. A través de la medición de la intensidad de la señal luminosa es posible cuantificar la concentración del complejo de interés en la muestra bajo análisis. Mediante esta metodología se pueden detectar en forma específica diferentes complejos en tan solo unos minutos, así como también usar diferentes tipos de NPs entre ellas NPs de oro. Más aún, es posible utilizar nanocomplejos de diferentes colores permitiendo desarrollar sistemas multiplexados capaces de detectar más de un complejo de interés. Con esta técnica, recientemente se ha reportado la detección de Vaspin, una proteína presente en la sangre que puede causar diabetes tipo 2, en concentraciones tan bajas como 35 pg/ml (Ali et al., 2017) 
FIGURA 5. Diagrama esquemático de una tira de flujo laminar (a), así como la fotografía de una tira con una línea de control positiva usando nanopartículas con conversión ascendente (b).

a)

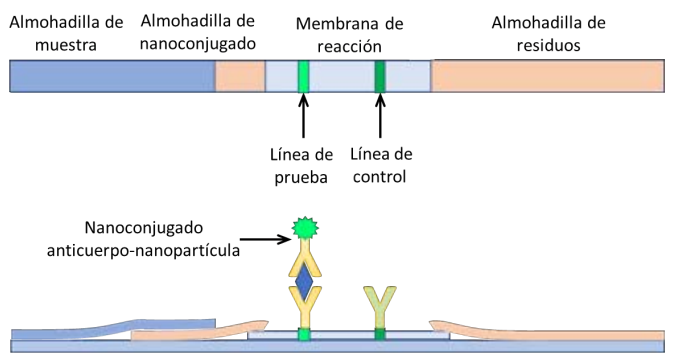

b)

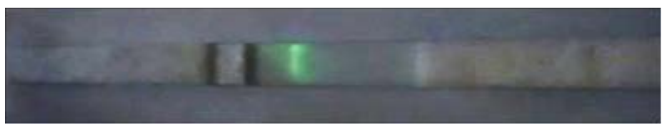

Fuente: Información detallada para el diseño de este tipo de tiras activas se puede encontrar en: Ali et al. (2017); Lian et al. (2017); He et al. (2016).

\section{Esparcimiento elástico de luz}

Como discutimos antes, el realce del esparcimiento de luz incidente sobre una NP de oro ha motivado el estudio de diferentes aproximaciones para el desarrollo de biosensores. En particular la síntesis de NPs terminadas en punta tipo estrella, incrementa el esparcimiento y recorre el plasmón de resonancia hacia el cercano infrarrojo (NIR) favoreciendo las aplicaciones biomédicas al alcanzar la primera ventana biológica (Colleen et al., 2006; Kumar et al., 2007; Xie et al., 2017). Además, presentan una depolarización de la luz 15 veces mayor con respecto a la obtenida por las NPs esféricas, lo que ha permitido implementar la técnica de microscopia de campo obscuro sensible a la polarización, como hemos reportado recientemente (figura 6), (Aaron, et al., 2008). La conjugación de NPs contra algún complejo de interés permite el desarrollo de biosensores de mayor sensibilidad y altamente selectivos, haciendo posible su uso en el monitoreo in-situ de biomoléculas como se discutirá más adelante.

\section{Dispersión Raman mejorada por efecto de superficie (SERS)}

Recientemente, las NPs tipo estrella han recobrado un mayor impulso para ser utilizadas en el diseño de sustratos SERS particularmente útil para la detección de analitos a concentraciones muy bajas (Kumar et al., 2007; Pérez-Mayen et al., 2015; Hiang Kwee Lee et al., 2019). En general, cuando se colocan NPs metálicas lo suficientemente cerca se traslapan los campos maximizándose en un punto que se conoce como punto caliente o hot spot que, de acuerdo con cálculos reportados, se localiza en el punto medio entre nanopartículas separadas alrededor de $2 \mathrm{~nm}$ (figura 7a), (Kumar et al., 2007). Este efecto es mayor para las 
Mundo Nano | ARTículos DE INVESTIGACIÓN | www.mundonano.unam.mx

13(24), 1e-24e, enero-junio 2020 | http://dx.doi.org/10.22201/ceiich.24485691e.2020.24.69618 Elder De la Rosa, Gonzalo Ramírez, Sandeep Panikar, Tanya Camacho, Pedro Salas, Tzarara López-Luke

FIGURA 6. Imágenes de microscopía electrónica de barrido de NPs de oro esféricas y tipo estrella.
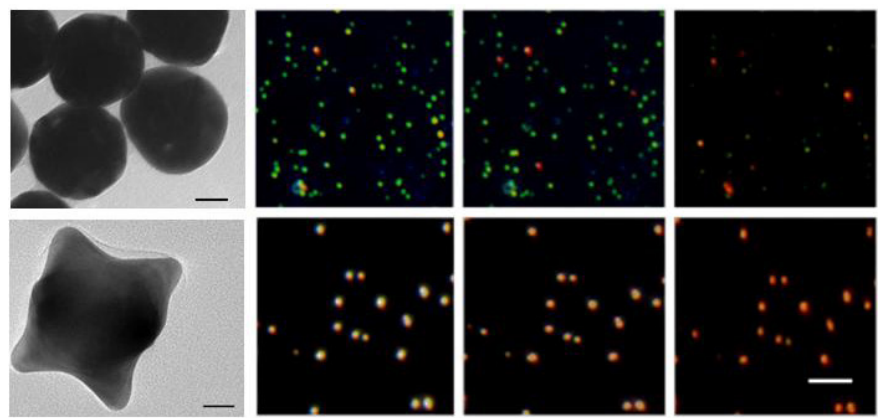

$n p$

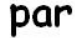

per

Nota: A la izquierda del panel superior e inferior, la barra de escala es de $20 \mathrm{~nm}$. Esparcimiento de luz de NPs de oro esféricas (panel superior) y tipo estrella (panel inferior) obtenido por microscopía de campo obscuro sensible a la polarización. El color rojo de la luz esparcida por NPs tipo estrella sugiere el corrimiento del plasmón de resonancia hacia el rojo. Observe que cuando el polarizador de entrada y salida están perpendiculares (per) el esparcimiento de las esferas se reduce drásticamente mientras que el correspondiente a las estrellas permanece bastante brillante. Cuando los polarizadores están paralelos (par) solo se observa una pequeña disminución de la luz esparcida en ambos casos. La barra de escala es $5 \mu \mathrm{m}$.

Fuente: Elaboración de los autores.

NPs que terminan en punta aprovechando el efecto nanoantena demostrado ampliamente teórica y experimentalmente (Kumar et al., 2007; Pérez-Mayen et al., 2015; Hiang Kwee Lee et al. 2019). Mejor aún, las NPs plasmónicas empleadas para el diseño de sustratos SERS tienen la capacidad de sintonización para estar en resonancia con el láser de excitación incrementando la dispersión de la señal. Estas características hacen del SERS una técnica inmejorable para la detección molecular a nivel de zeptomoles $\left(10^{-21}\right.$ moles) de concentración (Kumar et al., 2007). Con tal sensibilidad, se ha reportado la aplicación de la técnica SERS en el área biomédica, desde la detección de biomarcadores de cáncer, infecciones fúngicas, infecciones bacterianas, entre otras; también se ha empleado en muestras de fluidos corporales como saliva, plasma sanguíneo, suero y esputo, así como en la detección de fármacos libres, sin la necesidad de etiquetas o de moléculas reporteras (Panikar et al., 2019; Zukovskaja et al., 2019; PérezMayen et al., 2016; Ceja-Fdez. et al., 2014).

Sin embargo, el mayor problema es el diseño de sustratos con una distribución homogénea de los puntos calientes. La manera de mantener los puntos calientes es funcionalizando en forma apropiada las NPs para que mantengan la separación entre partículas alrededor de $2 \mathrm{~nm}$ y sin que interfieran en la aproximación del complejo que se desea medir. Gracias a esta aproximación hemos reportado factores de realce del orden de $10^{9}$ con nanoestrellas de oro, y $10^{12}$ cuando estas se depositan sobre una película de oro formando puntos calientes entre partícula-partícula y entre partícula-película. Con estas sensibilidades hemos medido concentraciones tan bajas como $10^{-22}$ moles en un 
área de $189 \mu \mathrm{m}^{2}$ lo cual equivale a 1 molécula cada $3 \mu \mathrm{m}^{2}$ (figura 7b), (PérezMayen, 2016). Más recientemente, hemos desarrollado sensores SERS basados en óxido de grafeno (GO) donde se anclan nanopartículas de oro tipo estrella funcionalizadas con L-cisteína para evitar el efecto de bloqueo de los puntos calientes por las proteínas de la sangre. El anclaje de las NPs de oro se logra mediante la activación de grupos carboxilos en la superficie del grafeno, usando el método de Hummers. Con esta técnica hemos logrado la detección en sangre de hasta $10^{-8} \mathrm{M}$ de paclitaxel y $10^{-9} \mathrm{M}$ de ciclofosfamida, medicamentos ampliamente utilizados en tratamientos contra cáncer abriendo una avenida para el desarrollo de una medicina personalizada (MP). La MP toma en cuenta que cada organismo reacciona y/o asimila un medicamento en forma diferente, por lo cual, conocer con mayor precisión la cantidad exacta de medicamento que asimila cada cuerpo ayuda a mejora la terapia y reducir los problemas toxicológicos. Un análisis detallado de la preparación de NPs de oro tipo estrella, su funcionalización, activación del grafeno, así como resultados en la detección de medicamentos se reporta en Panikar et al. (2019).

\section{Nanomedicina}

El uso de la nanotecnología en la medicina ha cobrado gran relevancia para la implementación de nuevas estrategias para la atención de diferentes problemas de salud que aquejan a la población. Sin duda alguna, el cáncer es uno de esos grandes problemas que están siendo estudiados (Riehemann et al., 2009; Shi et al., 2017; Lammers et al., 2011; Bai et al., 2015; Björnmalm et al., 2017). El cáncer, por su agresividad, incidencia, complejidad e incremen-

FIGURA 7. a) Representación esquemática de la generación de hot spot debido a la superposición de campo electromagnético entre dos nanopartículas que dan como resultado el realce de la señal Raman en la espectroscopía Raman mejorada en superficie. b) Señal Raman de la Rodamina B a diferentes concentraciones utilizando solamente NPs de oro tipo estrellas y combinada con una película de oro, se observa el efecto de la película de oro en el factor de realce y con ello una mayor sensibilidad.
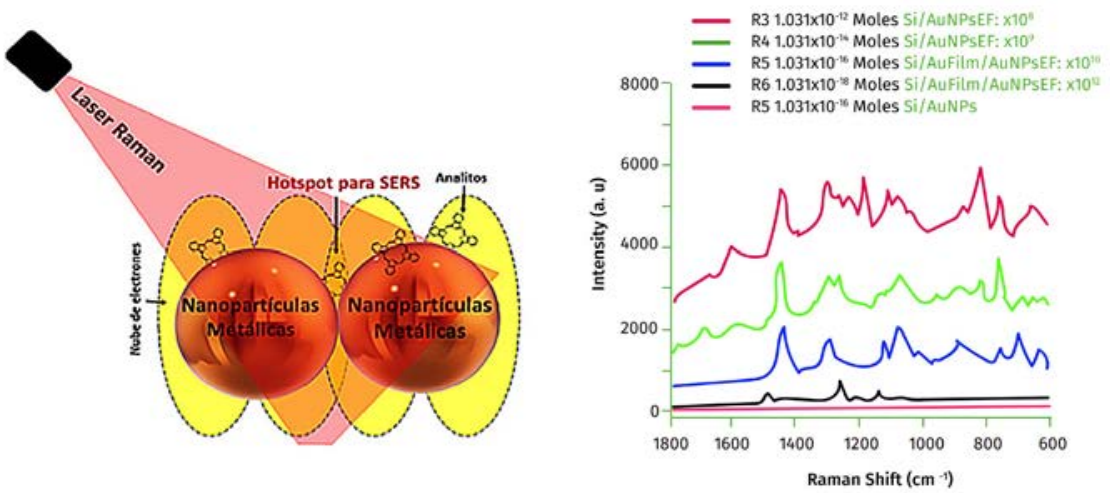

Fuente: La figura 7b fue tomada de Pérez-Mayen (2016). 
to en la fármaco-resistencia, requiere avances en la nanomedicina que permitan detectar y tratar de forma eficiente los mecanismos de desarrollo y metástasis del tumor. Gracias a la alta sensibilidad de las técnicas de detección desarrolladas y discutidas anteriormente, es posible detectar concentraciones muy bajas de proteínas sobre expresadas por algún tipo de cáncer permitiendo un diagnóstico temprano y mejorando las expectativas de éxito de la terapia aplicada. De la misma manera, se han venido desarrollando estrategias desde la nanotecnología para la terapia contra el cáncer que garanticen efectividad y reduzcan los efectos secundarios.

\section{Detección e imagen}

El aprovechamiento de las propiedades ópticas de los nanomateriales y su uso como agentes de contraste ha permitido desarrollar estrategias para la detección por imagenología o escaneo biológico de la presencia de tumores o células cancerígenas en etapas muy tempranas. Es una herramienta en desarrollo útil para evaluar de manera no invasiva la localización y progresión de enfermedades, los efectos de un medicamento o su comportamiento farmacocinético, así como la identificación de biomarcadores de enfermedades. Además de sus propiedades funcionales, para que los nanomateriales puedan ser utilizados como agentes de contraste y, en general, para aplicaciones biomédicas, existen al menos tres requerimientos principales: i) excelente biocompatibilidad; ii) buena dispersión en soluciones acuosas, y, iii) la presencia de grupos funcionales en la superficie que permitan la conjugación con moléculas biológicas activas o moléculas de interés, o la repulsión con las mismas, por ejemplo, para evitar su detección por el sistema inmunológico. El requisito fundamental para el escaneo biológico y la detección temprana es garantizar la selectividad de los agentes de contraste. Esto se logra conjugándolos con anticuerpos, péptidos u oligonucleótidos específicos, de manera que se adhieran solamente a los biomarcadores definidos (Lécuyer et al., 2016; Ramírez-García et al., 2015).

En nuestro grupo hemos utilizado puntos cuánticos (QDs), nanopartículas fluorescentes por conversión ascendente (UCNPs) y nanopartículas de oro (NPAu) para marcar células cancerígenas (figura 8). Además de detectar la presencia de células cancerígenas y/o presencia de proteínas sobre expresadas por ese tipo de cáncer particular, los agentes de contraste nos permiten evaluar los mecanismos de internalización celular y delimitar el área dañada sin afectar las células sanas. La figura 8a muestra células sanas obtenidas directamente de la mucosa oral marcadas con QDs de CdTe sin conjugar, de 4 nm de diámetro, dispersas en agua, que se internalizan y marcan el núcleo y el citoplasma de la célula. El proceso de internalización con NPs no conjugadas se logra controlando el tiempo de incubación; sin embargo, cuando las NPs son muy pequeñas como los QDs la internalización se facilita como puede observarse en la figura 8a. Para un análisis detallado de la síntesis de puntos cuánticos y su uso en el marcado de células se recomienda ver la referencia (Cepeda-Pérez et al., 2016). 
La figura 8c muestra células SKBR-3 que sobre expresan la proteína HER2 (Human epidermal grow factor receptor 2 o receptor 2 para el factor de crecimiento epidérmico humano), marcadas con nanopartículas de $\mathrm{NaYF}_{4}: \mathrm{Yb}$,Er conjugadas de manera covalente con el anticuerpo monoclonal Herceptin, específico para el receptor HER2-positivo presente en la superficie de dichas células de cáncer de mama. Es posible observar la presencia de los nanoconjugados en el citoplasma, mientras que el núcleo ha sido marcado de manera simultánea con el colorante Hoetchst para facilitar el contraste. La fluorescencia observada al ser excitadas a $980 \mathrm{~nm}$ indica la presencia del nanocomplejo conjugado y con ello la presencia de la proteína HER2, señalando que la célula está dañada. Esta técnica, además de detectar la presencia de la proteína sobre expresada, indica también el área dañada lo cual permite una acción localizada y de mayor precisión de un proceso de terapia reduciendo los efectos no deseados al afectar células sanas. La conjugación de las NPs con anticuerpos garantiza, además de la selectividad, la detección de bajas concentraciones (en el rango de nano a pico gramos) de la proteína receptora sobre expresada por el cáncer. El proceso de síntesis y conjugación de las NPs se muestra en forma detallada en la referencia (Ramírez-García et al., 2018). La figura 8b muestra células de cáncer de piel A-431 que sobre expresan la proteína EGFR del orden de $10^{6}$ moléculas por célula, marcadas con NPs de oro tipo estrella conjugadas con anti-EGFR mezcladas con células sin marcar en una razón 1:1. Las NPs de oro tipo estrella fueron sintetizadas mediante la reducción de acido cloroaurico con acido ascórbico en agua a $0{ }^{\circ} \mathrm{C}$. Posteriormente, se conjugan con anticuerpos monoclonales y se dispersan en agua para incubarse con las células. Una descripción detallada de la síntesis y conjugación de las NPs se puede encontrar en la referencia (Aaron et al., 2008). La imagen en la figura $8 \mathrm{~b}$ fue obtenida con la técnica de microscopía de campo oscuro con el polarizador y analizador a 90 grados. Se observan claramente las células marcadas y en forma muy débil las células no marcadas, lo que muestra el efecto de la depolarización de la luz por parte de las NPs tipo estrella. La alta sensibilidad de esta técnica sugiere su uso en la detección de eventos de una sola molécula en células, que sería equivalente a la detección de una sola NP adherida a una sola molécula. El recuadro interior en la figura 8b muestra la internalización de tres NPs de oro tipo estrella indicando el potencial de la técnica para la identificación de una sola molécula (Aaron et al., 2008). Estos resultados sugieren un gran potencial para la detección de bajas concentraciones de proteína sobre expresada lo que puede coadyuvar a la detección temprana de cáncer.

En el ámbito terapéutico, los nanomateriales han sido ampliamente utilizados en terapia fotodinámica, terapia fototérmica y como acarreadores de fármacos o nanovectores (Huang et al., 2006; Gobin et al., 2007; RamírezGarcía et al., 2018; Zhou et al., 2017). Más recientemente se han venido desarrollando complejos teranósticos que combinan detección, imagen y terapia permitiendo también monitorear el efecto de la misma Lammers et al., 2011; Bai et al., 2015; Gobin et al., 2007; Ramírez-García et al., 2018; Zhou 
Mundo Nano | ARTículos DE INVESTIGACIÓN | www.mundonano.unam.mx 13(24), 1e-24e, enero-junio 2020 | http://dx.doi.org/10.22201/ceiich.24485691e.2020.24.69618 Elder De la Rosa, Gonzalo Ramírez, Sandeep Panikar, Tanya Camacho, Pedro Salas, Tzarara López-Luke

FIGURA 8. a) Células sanas obtenidas de la mucosa oral marcadas con puntos cuánticos de CdTe sin conjugar, imagen tomada con microscopio de epifluorescencia, la barra de escala es $5 \mu \mathrm{m}$; b) NPs de oro tipo estrellas conjugadas para marcar células cancerígenas A-431 obtenida por microscopía óptica sensible a la polarización (el polarizador de entrada y el de salida están perpendiculares; los círculos blancos indican células sanas que no fueron marcadas. El recuadro muestra la internalización celular de NPs de oro reportado anteriormente (Aaron et al., 2008); c) microscopía confocal de células SKBR3 (cáncer de mama HER2 positiva) con su núcleo marcado con el colorante Hoecht (azul), y nanoconjugados $\mathrm{NaYF}_{4}: \mathrm{Yb}, \mathrm{Er}$-Herceptin (rojo).
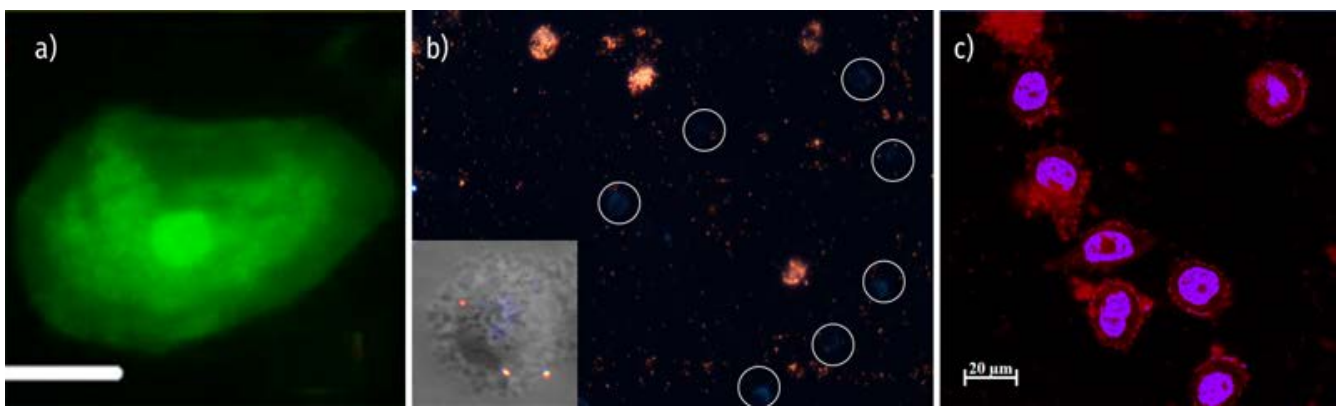

Fuente: Elaboración de los autores.

et al., 2017). Estos complejos prometen la implementación de una medicina personalizada y preventiva aprovechando la alta sensibilidad de las técnicas de detección ya discutidas.

\section{Terapia fotodinámica}

La terapia fotodinámica (TFD) es una estrategia médica para el tratamiento del cáncer, considerada mínimamente tóxica e invasiva, capaz de reducir los efectos secundarios causados, por ejemplo, por tratamientos quirúrgicos, radioterapia o quimioterapia. Involucra al menos tres elementos: i) luz de longitud de onda adecuada; ii) oxígeno, y, iii) un fotosensibilizador. Una vez que se excita al fotosensibilizador este transfiere energía al oxígeno triplete que lo convierte en oxígeno singulete $\left({ }^{3} \mathrm{O}_{2}+h v_{T R} \rightarrow{ }^{1} \mathrm{O}_{2}\right)$ y se generan, además, pares electrón-hueco que reaccionan ya sea reduciendo el oxígeno circundante $\left(\mathrm{e}^{-}+\mathrm{O}_{2} \rightarrow{ }^{\bullet} \mathrm{O}_{2}^{-} y^{\bullet} \mathrm{O}_{2}^{-}+2 \mathrm{OH}+\mathrm{H}^{+} \rightarrow \mathrm{H}_{2} \mathrm{O}_{2}+\mathrm{O}_{2}\right)$ u oxidando el agua periférica $\left(h^{+}+\mathrm{H}_{2} \mathrm{O} \rightarrow \mathrm{H}^{+}+\mathrm{OH}^{-}\right.$y $\left.2 \mathrm{~h}^{+}+2 \mathrm{H}_{2} \mathrm{O} \rightarrow \mathrm{H}_{2} \mathrm{O}_{2}+2 \mathrm{H}^{+}\right)$. El resultado total es la formación de especies reactivas de oxígeno (ROS), oxígeno singulete, super óxido, hidroxilo, peróxido de hidrógeno, siendo estos los responsables del estrés oxidativo y capaces de inducir la muerte celular de las células malignas de manera sitio-dirigida. Sin embargo, la aplicación de la TFD no ha podido realizarse de manera efectiva debido a la frecuente hidrofobicidad de los fotosensibilizadores, su degradabilidad química y óptica, y la limitada capacidad de la luz para penetrar a través de los tejidos o la piel hasta llegar al fotosensibilizador y desencadenar su acción terapéutica (Lan et al., 2019).

Recientemente, la TFD ha emergido como una opción muy atractiva al conjugar fotosensibilizadores con diversos nanomateriales a fin de sobreponerse a las limitaciones descritas anteriormente. Estas NPs actúan simple- 
mente como vehículos para la entrega de la carga terapéutica en el sitio de acción, o como transductores de energía capaces de modular la intensidad y longitud de onda de la luz usada para la excitación de los fotosensibilizadores (Lécuyer et al., 2016; Ramírez-García et al., 2015 y 2018; Lan et al., 2019).

En nuestro grupo, hemos utilizado NPs con emisión por conversión ascendente (UCNPs) como transductores de energía, gracias a su capacidad de convertir la luz infrarroja en emisiones en las regiones ultravioleta o visible vía la absorción de múltiples fotones. La energía de estas emisiones es capaz de activar los fotosensibilizadores y con ello la acción terapéutica de la terapia fotodinámica. En una primera aproximación, usamos NPs de $\mathrm{NaYF}_{4}: \mathrm{Yb}, \mathrm{Er}$ con emisión por conversión ascendente en las regiones roja y verde del espectro electromagnético, conjugadas mediante enlaces covalentes con una ftalocianina de zinc que actúa como fotosensibilizador. Después de excitar a $975 \mathrm{~nm}$, las UCNPs son capaces de transferir la banda de emisión roja a las ftalocianinas por medio de FRET, desencadenando la producción de especies reactivas de oxígeno (ROS). Para garantizar la selectividad, se conjuga el complejo UNCPs-ftalocianinas con el anticuerpo monoclonal Trastuzumab permitiendo al nanocompuesto interactuar de manera específica con células de cáncer de mama HER2-positivo, una clase de cáncer particularmente agresiva. Con ello se logró disminuir la viabilidad celular hasta $21 \%$ después de administrar $200 \mu \mathrm{g} / \mathrm{mL}$ de immunoconjugados a las células y exponerlas durante 5 minutos a la luz infrarroja a una densidad de potencia de $0.71 \mathrm{~W} / \mathrm{cm}^{2}$. Además, la banda de emisión roja se encuentra en la región de la ventana de transparencia de tejidos, permitiendo el escaneo biológico o la posible obtención de imágenes a través de la piel (Ramírez-García et al., 2018).

En una segunda aproximación utilizamos nanocomplejos con estructura núcleo@coraza para producir la terapia fotodinámica. En este caso, utilizamos como núcleo NPs de $\mathrm{NaYF}_{4}: \mathrm{Yb}$,Tm cubierta por una capa de unos cuantos nanómetros de una mezcla de $\mathrm{TiO}_{2}-\mathrm{ZrO}_{2}$ que funciona como fotosensibilizador. Este complejo fue conjugado con el mismo anticuerpo monoclonal Trastuzumab para darles selectividad ante células HER2-positivas de cáncer de mama. En este nanoconjugado se optimizó la concentración de titania y zirconia para aumentar de manera simultánea la intensidad de emisión del $\mathrm{Tm}^{3+}$ en el cercano infrarrojo $(801 \mathrm{~nm}$ ) que se utiliza para detección e imagen de las células y la producción de ROS de diferente naturaleza después de la transferencia de energía proveniente de la banda de emisión ultravioleta de las UCNPs. En condiciones similares al experimento con las ftalocianinas, se logró la reducción de la viabilidad celular de las células de cáncer de mama hasta el $12 \%$ con una carga de $400 \mu \mathrm{g} / \mathrm{mL}$ de immunoconjugados. La presencia de un fotosensibilizador inorgánico en forma de coraza brinda, además, excelente estabilidad óptica y química (Ramírez-García et al., 2019). En todos los casos la viabilidad celular se midió utilizando el kit azul-alamar (alamar blue) que es un ensayo bien establecido que funciona mediante el monitoreo y cuantificación de los niveles de fluorescencia como respuesta a 
procesos de oxidación-reducción (REDOX) como resultado de una reducción del metabolismo celular. La fluorescencia se midió utilizando el equipo Cytation 5 de Bioteck.

\section{Terapia fototérmica}

El aumento inducido de la temperatura mediante la incidencia de luz, y su uso como método de terapia para inducir la muerte celular es conocido como hipertermia o terapia fototérmica. De manera similar a los nanocomplejos para TFD, las UCNPs también pueden ser conjugadas con agentes fototérmicos para provocar el aumento local de temperatura, desencadenando la inducción de la apoptosis de las células malignas en el sitio de la incidencia de luz (Ruichan Lv et al., 2017; Li et al., 2018). En nuestro laboratorio hemos creado un nanocomplejo de $\mathrm{NaYF}_{4}: \mathrm{Yb}$,Er con emisiones en las regiones verde y roja, conjugadas con nanopartículas de oro de tamaño inferior a los $10 \mathrm{~nm}$. La luz emitida por las UCNPs en los nanocomplejos es usada de manera simultánea para la excitación del plasmón de resonancia de las nanopartículas de oro (banda verde), el sensado in situ de la temperatura (nanotermómetro), y como agente de contraste para el escaneo y detección de células cancerosas mediante luminiscencia (banda roja). Se logró un aumento paulatino de la temperatura (hasta $42.2^{\circ} \mathrm{C}$ en 5 minutos), logrando la reducción de la viabilidad celular por debajo del $40 \%$ con condiciones similares a las usadas en TFD. Se estima que dicho valor puede disminuir aún más al incidir la luz infrarroja para la excitación de las UCNPS durante periodos más largos. Un resultado importante de este experimento es el uso simultáneo del nanotermómetro para monitorear y mantener la temperatura dentro del rango clínico de aplicación $\left(40-45^{\circ} \mathrm{C}\right)$ con una precisión de $\pm 0.5^{\circ} \mathrm{C}$, induciendo la muerte celular de las células cancerosas, sin afectar las células sanas (Ramírez-García et al., 2019b).

Otro tipo de nanocompuestos para terapia fototérmica fue desarrollado en nuestro grupo mediante el dopaje de nanopartículas de $\mathrm{Fe}_{3} \mathrm{O}_{4}$ con lantánidos, logrando la integración simultánea de las propiedades magnéticas y de emisión por conversión ascendente en compuestos de tipo $\mathrm{Fe}_{3} \mathrm{O}_{4}: \mathrm{Yb}$, Er. De manera inesperada, se observó un aumento espontáneo de la temperatura de las nanopartículas al irradiar con luz infrarroja. Se estima que estas nanopartículas podrán ser utilizadas también para el escaneo biológico y terapia fotodinámica de manera simultánea (Ibarra-Sánchez et al., 2018).

\section{Teranóstica}

La teranóstica es un campo de reciente creación en la medicina que combina capacidades de diagnóstico y de terapia en una sola herramienta terapéutica. Adicionalmente, implica el monitoreo de la respuesta del paciente a la terapia aplicada logrando mejorar la calidad del tratamiento. El acoplamiento de materiales con diversas propiedades fisicoquímicas como la luminiscencia, magnetismo, termo-responsividad, capacidad de acarreamiento de fármacos y resistencia mecánica e inocuidad pueden brindar nuevas herramientas te- 
ranósticas, permitiendo monitorear el progreso de la enfermedad mientras se induce un efecto terapéutico. En nuestro grupo trabajamos en el diseño de estos complejos teranósticos combinando NPs altamente sensibles a la detección e imagen de complejos de interés, capaces de inducir muerte celular por efecto fototérmico, terapia fotodinámica o por la entrega dirigida de medicamentos. Aunque hay varios esquemas para formar estos nanocomplejos, nosotros diseñamos nanocomplejos a base de sílica mesoporosa, polímeros y a base de lípidos. Nuestra investigación continúa arrojando resultados muy interesantes. Recientemente hemos logrado avances muy prometedores combinando terapia fotodinámica y entrega dirigida de fármacos reduciendo la viabilidad celular por debajo del $10 \%$ como resultado del efecto sinérgico entre las dos técnicas terapéuticas en células cancerígenas HER2-positivas, resultados que han sido enviados para su publicación.

\section{Conclusión}

La nanotecnología está impactando todas las áreas de la actividad humana. Se están diseñando nuevos dispositivos que definirán nuestro estilo de vida futura, especialmente en las áreas de la comunicación y de la salud. Gracias a los nanomateriales, se están diseñando dispositivos para la detección y diagnóstico de enfermedades que no requerirán la ayuda médica y que no requieren grandes equipos. Este proceso se ha venido acelerando al agregar a estos nuevos dispositivos inteligencia artificial que, combinados, seguramente lograrán muy pronto que la detección y el diagnóstico de enfermedades se haga desde un teléfono inteligente. La inclusión de complejos teranósticos promete una gran herramienta para el combate a enfermedades de gran importancia para la humanidad. Esta relativa facilidad de detección, diagnóstico y terapia está delineando el camino para diseñar una medicina que no solo sea preventiva sino personalizada y de bajo costo, verdaderamente al alcance de todos. Sin embargo, es importante realizar estudios profundos sobre el impacto toxicológico en organismos vivos tanto por el uso de nanocomplejos en procesos terapéuticos como la exposición involuntaria de NP.

\section{Referencias}

Aaron, Jesse; Elder De La Rosa, Kort Travis, Nathan Harrison, Justin Burt, Miguel José-Yacamán, Konstantin Sokolov. (2008). Polarization microscopy with stellated gold nanoparticles for robust, in-situ monitoring of biomolecules. Optic express, 16: 2153-2167. http://dx.doi.org/10.1364/OE.16.002153

Ali, Muhsin; Memoon Sajid, Muhammad Asad Ullah Khalid, Soo Wan Kim, Jong Hwan Lim, Dongeun Huh, Kyung Hyun Choi. (2020). A fluorescent lateral flow biosensor for the quantitative detection of Vaspin using upconverting nanoparticles. Spectrochimica Acta Part A, 226: 117610.

http://dx.doi.org/10.1016/j.saa.2019.117610 
Ament, Irene; Janak Prasad, Andreas Henkel, Sebastian Schmachtel, Carsten Sönnichsen. (2012). Single unlabeled protein detection on individual plasmonic nanoparticles. Nano Lett., 12: 1092-1095. http://dx.doi.org/10.1021/nl204496g

Bai, R. G.; K. Muthoosamy, S. Manickam. (2015). Nanomedicine in theranostics, nanotechnology applications for tissue engineering, cap. 12, 195-213.

http://dx.doi.org/10.2147/IJN.S153758

Bauch, Martin; Koji Toma, Mana Toma, Qingwen Zhang, Jakub Dostalek. (2014). Plasmon-enhanced fluorescence biosensors: a review. Plasmonic, 9: 781-799. http://dx.doi.org/10.1007/s11468-013-9660-5

Bera, Debasis; Lei Qian, Teng-Kuan Tseng y Paul H. Holloway. (2010). Quantum dots and their multimodal applications: a review. Materials, 3: 2260-2345;

http://dx.doi.org/10.3390/ma3042260

Björnmalm, Mattias; Kristofer J. Thurecht, Michael Michael, Andrew M. Scott y Frank Caruso. (2017). Bridging bio-nano science and cancer nanomedicine. ACS Nano, 11: 9594-9613. http://dx.doi.org/10.1021/acsnano.7b04855

Burda, Clemens; Xiaobo Chen, Radha Narayanan y Mostafa A. El-Sayed. (2005). Chemistry and properties of nanocrystals of different shapes. Chem. Rev., 105: 1025-1102. http://dx.doi.org/10.1021/cr030063a

Ceja-Fdez, A.; T. López-Luke, A. Torres-Castro, D. A. Wheeler, J. Z. Zhang y E. De la Rosa. (2014). Glucose detection using SERS with multi-branched gold nanostructures in aqueous médium. RSC Advances, 4: 59233-59241.

http://dx.doi.org/10.1039/C4RA11055B

Cepeda-Pérez, Elisa; Iris Aguilar-Hernández, Tzarara López-Luke, Valeria Piazza, Ramón Carriles, Nancy Ornelas-Soto, Elder De la Rosa. (2016). Interaction of TGA@CdTe quantum dots with an extracellular matrix of Haematococcus pluvialis microalgae detected using surface-enhanced Raman spectroscopy (SERS). Appl. Spectroscopy, 70: 1561-1572. http://dx.doi.org/10.1177/0003702816654076

Cherenack, Kunigunde y Liesbeth van Pieterson. (2012). Smart textiles: Challenges and oportunities. J. Appl. Phys., 112: 091301.

http://dx.doi.org/10.1063/1.4742728

Choi, Suji; Hyunjae Lee, Roozbeh Ghaffari, Taeghwan Hyeon, Dae-Hyeong Kim. (2016). Recent advances in flexible and stretchable bio-electronic devices integrated with nanomaterials. Adv. Funct. Mat., 28: 4203-4218;

http://dx.doi.org/10.1002/adma.201504150

Chorsi, Hamid T.; Youngkyu Lee, Andrea Alu y Johm X. J. Zhang. (2017). Tunable plasmonic substrate with ultrahigh Q-factor resonance. Sci. Reports, 7: 15985; http://dx.doi.org/10.1038/s41598-017-16288-3

Colleen, L.; Nehl Hongwei, Liao Jason, H. Hafner. (2006). Optical properties of starshaped gold nanoparticles. Nano Lett., 6: 683-688.

http://dx.doi.org/10.1021/nl052409y

Das, B. B. y Arkadeep Mitra. 2014). Nanomaterials for construction engineering-A review. Int. Journal of Materials and Manufacturing, 2: 41-46.

http://dx.doi.org/10.7763/IJMMM.2014.V2.96

De la Rosa-Cruz, Elder; L. A. Díaz-Torres, P. Salas, R. A. Rodríguez, G. A. Kumar, M. 
A. Meneses, J. F. Mosino, J. M. Hernández, O. Barbosa-García. (2003). Luminescent properties and energy transfer in $\mathrm{ZrO}_{2}: \mathrm{Sm}^{3+}$ nanocrystals. J. Appl. Phys., 94: 3509-3515. http://dx.doi.org/10.1063/1.1599960

Dong, Hao; Ling-Dong Sun y Chun-Hua Yan. (2015). Energy transfer in lanthanide upconverting studies for extended optical applications. Chem. Soc. Rev., 44: 1608-1634. http://dx.doi.org/10.1039/C4CS00188E

Giust, Davide; María Isabel Lucío, Afaf H. El-Sagheer, Tom Brown, Lorraine E. Williams, Otto L. Muskens, Antonios G. Kanaras. (2018). Graphene oxide-upconversion nanoparticle based portable sensors for assessing nutritional deficiencies in crops. ACS Nano, 12: 6273-6279. http://dx.doi.org/10.1021/ acsnano.8b03261

Gnach, Anna y Artur Bednarkiewicz. (2012). Lanthanide-doped up-converting nanoparticles: Merits and challenges. Nanotoday, 7: 532-563. http://dx.doi.org/10.1016/j.nantod.2012.10.006

GobinMin, André M.; Ho Lee Naomi, J. Halas William, D. James Rebekah, A. Drezek Jennifer, L. West. (2007). Near-infrared resonant nanoshells for combined optical imaging and photothermal cancer therapy. Nano Lett. 7: 1929-1934. http://dx.doi.org/10.1021/nl070610y

$\mathrm{Gu}$, B. y Zhang, Q. (2018) Recent advances on functionalized upconversion nanoparticles for detection of small molecules and ions in biosystems. Adv. Sci., 9: 1700690. http://dx.doi.org/10.1002/advs.201700609

He, Zheng-Xin; Lan-Chun Shi, Xiang-Yang Ran, Wei Li, Xian-Ling Wang y Fu-Kun Wang. (2016). Development of a lateral flow immunoassay for the rapid diagnosis of invasive candidiasis. Front. in Microbiol., 7: 1451.

http://dx.doi.org/10.3389/fmicb.2016.01451

Hildebrand, Niko. (2011). Biofunctional quantum dots: controlled conjugation for multiplexed biosensors. ACS Nano, 5: 5286-5290.

http://dx.doi.org/10.1021/nn2023123

Huang, Xiaohua; Ivan H. El-Sayed, Wei Qian, Mostafa A. El-Sayed. (2006). Cancer cell imaging and photothermal therapy in the near-infrared region by using gold nanorods. J. Am. Chem. Soc., 128: 2115-2120;

http://dx.doi.org/10.1021/ja057254a

Ibarra-Sánchez, José de Jesús; Tzarara López-Luke, Gonzalo Ramírez-García, Siraj Sidhik, Teodoro Córdova-Fraga, José de Jesús Bernal-Alvarado, M. Eduardo Cano, Alejandro Torres-Castro, Elder De la Rosa. (2018). Synthesis and characterization of $\mathrm{Fe}_{3} \mathrm{O}_{4}: \mathrm{Yb}^{3+}$ : $\mathrm{Er}^{3+}$ nanoparticles with magnetic and optical properties for hyperthermia applications. J. of Magnetism and Magnetic Materials, 465: 406-411. http://dx.doi.org/10.1016/j.jmmm.2018.05.091

Kneipp, Janina; Harald Kneipp y Katrin Kneipp. (2008). SERS — a single-molecule and nanoscale tool for bioanalytics. Chem. Soc. Rev., 37: 1052-1060. h t tp:// dx.doi.org/10.1039/B708459P

Kumar, Pandian Senthil; Isabel Pastoriza-Santos, Benito Rodríguez-González, F. Javier García de Abajo y Luis M. Liz-Marzán. (2007). High-yield synthesis and optical response of gold nanostars. Nanotechnology, 19: 015606. 
http://dx.doi.org/10.1088/0957-4484/19/01/015606

Kumar, Sandeep; Monika Nehra, Akash Deep, Deepak Kedia, Neeraj Dilbaghi, KiHyun Kim. (2017). Quantum sized nanomaterials for solar cell applications. Renew. and Sust. Energy, 37: 821-839;

http://dx.doi.org/10.1016/j.rser.2017.01.172

Lammers, Twan; Silvio Aime, Wim E. Hennink, Gert Storm, Fabian Kiessling. (2011). Theranostic nanomedicine. Acc. Chem. Res., 44: 1029-1038.

http://dx.doi.org/10.1021/ar200019c

Lan, Minhuan; Shaojing Zhao, Weimin Liu, Chun-Sing Lee, Wenjun Zhang, Pengfei Wang. (2019). Photosensitizers for photodynamic therapy. Advanced Healthcare Materials, 8: 1900132. http://dx.doi.org/10.1002/adhm.201900132

Lauterwasser, Christoph (ed.) (2005). Small sizes that matter: Opportunities and risk of nanotechnologies, report in cooperation with OECD International Futures Program. OECD, Allianz AG. http://www.oecd.org/science/nanosafety/37770473.pdf

Lécuyer, Thomas; Eliott Teston, Gonzalo Ramírez-García, Thomas Maldiney, Bruno Viana, Johanne Seguin, Nathalie Mignet, Daniel Scherman, Cyrille Richard. (2016). Chemically engineered persistent luminescence nanoprobes for bioimaging. Theranostic, 6: 2488-2524. http://dx.doi.org/10.7150/thno.16589

Lee, Hiang Kwee; Yih Hong Lee, Charlynn Sher Lin Koh, Gia Chuong Phan-Quang, Xuemei Han, Chee Leng Lay, Howard Yi Fan Sim, Ya-Chuan Kao, Qi An y Xing Yi Ling. (2019). Designing surface-enhanced Raman scattering (SERS) platforms beyond hotspot engineering: emerging opportunities in analyte manipulations and hybrid materials. Chem. Soc. Rev., 48: 731-756.

http://dx.doi.org/10.1039/C7CS00786H

Li, Ming; Scott K. Cushing y Niangiang Wu. (2015). Plasmon-enhanced optical sensors: a review. Analyst, 140: 386-406. http://dx.doi.org/10.1039/C4AN01079E

Li, Po; Yue Yan, Binlong Chen, Pan Zhang, Siling Wang, Jing Zhou, Haiming Fan, Yiguang Wang y Xiaonan Huang. (2018). Lanthanide-doped upconversion nanoparticles complexed with nano-oxide graphene used for upconversion fluorescence imaging and photothermal therapy. Biomater. Sci., 6: 877-884.

http://dx.doi.org/10.1039/c7bm01113j

Lian, Zhiqin; Xiaochen Wang, Wei Zhu, Pingping Zhang, Yongxin Yang, Chongyun Sun, Junjie Zhang, Xinrui Wang, Zheng Xu, Yong Zhao, Ruifu Yang, Suling Zhao, Lei Zhou. (2017). Upconversion nanocrystals mediated lateral-flow nanoplatform for in vitro detection. Appl. Mater. Interfaces, 9: 3497-3504.

http://dx.doi.org/10.1021/acsami.6b14906

Lv, Ruichan; Depeng Wang, Liyang Xiao, Guanying Chen, Jun Xia y Paras N. Prasad. (2017). Stable ICG-loaded upconversion nanoparticles: silica core/shell theranostic nanoplatform for dual-modal upconversion and photoacoustic imaging together with photothermal therapy. Scientific Reports, 7: 15753.

http://dx.doi.org/10.1038/s41598-017-16016-x

Mya Khin, Mya; A. Sreekumaran Nair, V. Jagadeesh Babu, Rajendiran Murugan y Seeram Ramakrishna. (2012). A review on nanomaterials for envioromental remediation. Energy Environ. Sci., 5: 8075-8109; 
http://dx.doi.org/10.1039/C2EE21818F

Noguez, Cecilia. (2007). Surface plasmons on metal nanoparticles: The influence of shape and physical environment. J. Phys. Chem. C., 111: 3806-3819.

http://dx.doi.org/10.1021/jp066539m

Oliverio, Manuela; Sara Perotto, Gabriele C. Messina, Laura Lovato, Francesco De Angelis. (2017). Chemical functionalization of plasmonic surface biosensors: a tutorial review on issues, strategies, and costs. Appl. Mater. Interfaces, 9: 29394-29411; http://dx.doi.org/10.1021/acsami.7b01583

Panikar, S. S.; G. Ramírez-García, S. Sidhik, T. López-Luke, C. Rodríguez-González, I. H. Ciapara, P. S. Castillo, T. Camacho-Villegas y E. De la Rosa. (2019). Ultrasensitive SERS substrate for label-free therapeutic-drug monitoring of paclitaxel and cyclophosphamide in blood serum. Analytical Chemistry, 91: 2100-2111. http://dx.doi.org/10.1021/acs.analchem.8b04523

Pérez-Mayen, L. (2016). SERS substrate with gold nanoparticles functionalized to detect specific analytes. Tesis de doctorado. CIO. http://cio.repositorioinstitucional. $\mathrm{mx} /$ jspui/bitstream/1002/415/1/16803.pdf

Pérez-Mayen, L.; J. Oliva, P. Salas y E. De la Rosa. (2016). Nanomolar detection of glucose using SERS substrates fabricated with albumin coated gold nanoparticles. Nanoscale, 8: 11862-11869. http://dx.doi.org/10.1039/c6nr00163g

Pérez-Mayen, Leonardo; Jorge Oliva, Alejandro Torres-Castro y Elder De la Rosa. (2015). SERS substrates fabricated with star-like gold nanoparticles for zeptomole detection of analytes. Nanoscale, 7: 10249-10258.

http://dx.doi.org/10.1039/c5nr02004b

Pissuwan, Dakrong; Stella M. Valenzuela, Michael B. Cortie. (2006). Therapeutic possibilities of plasmonically heated gold nanoparticles. Trends in biotchnology, 24: 62-67. http://dx.doi.org/10.1016/j.tibtech.2005.12.004

Pitkethly, Michael J. (2004). Nanomaterials - the driving forcé. Materials Today, 7: 20-29. http://dx.doi.org/10.1016/S1369-7021(04)00627-3

Pu, Yuan; Fuhong Cai, Dan Wang, OrcidJie-Xin Wang, OrcidJian-Feng Chen. (2018). Colloidal synthesis of semiconductor quantum dots toward large-scale production: a review. Ind. Eng. Chem. Res., 57: 1790-1802.

http://dx.doi.org/10.1021/acs.iecr.7b04836

Ramírez-García, G.; Miguel Ángel Honorato-Colin, Elder De la Rosa, Tzarara LópezLuke, Sandeep S. Panikar, José de Jesús Ibarra-Sánchez, Valeria Piazza. (2019). Theranostic nanocomplex of gold-decorated upconversion nanoparticles for optical imaging and temperature-controlled photothermal therapy. J. Photochemistry and Photobiology A: Chemistry, 384: 112053.

http://dx.doi.org/10.1016/j.jphotochem.2019.112053

Ramírez-García, G.; d’Orlyé F, Gutiérrez-Granados, Martínez-Alfaro, Mignet N., Richard C., Varenne A. (2015). Functionalization and characterization of persistent luminescence nanoparticles by dynamic light scattering, laser Doppler and capillary electrophoresis. Colloids Surf. B Biointerfaces, 1: 272-281.

http://dx.doi.org/10.1016/j.colsurfb.2015.09.02

Ramírez-García, G.; Elder De la Rosa, Tzarara López-Luke, Sandeep S. Panikar, Pedro 
Salas. (2019). Controlling trapping states on selective theranostic core@shell (NaYF4:Yb,Tm@TiO2-ZrO2) nanocomplexes for enhanced NIR-activated photodynamic therapy against breast cancer cells. Dalton Trans., 48: 9962-9973. http://dx.doi.org/10.1039/C9DT00482C

Ramírez-García, G.; Sandeep S. Panikar, Tzarara López-Luke, Valeria Piazza, Miguel Angel Honorato-Colin, Tanya Camacho-Villegas, Rodolfo Hernández-Gutiérrez y Elder De la Rosa. (2018). An immunoconjugated up-conversion nanocomplex for selective imaging and photodynamic therapy against HER2-positive breast cancer. Nanoscale, 10: 10154-10165; http://dx.doi.org/10.1039/c8nr01512k

Riehemann, Kristina; Stefan W. Schneider, Thomas A. Luger, Biana Godin, Mauro Ferrari, Harald Fuchs. (2009). Nanomedicine-Challenge and perspectives. Angewandte Chemie, 48: 872-897; http://dx.doi.org/10.1002/anie.200802585

Rowe, Chris A.; Leonard M. Tender, Mark J. Feldstein, Joel P. Golden, Stephanie B. Scruggs, Brian D. MacCraith, John J. Cras y Frances S. Ligler. (1999). Array biosensor for simultaneous identification of bacterial, viral, and protein analytes. Anal. Chem., 71: 3846-3852; http://dx.doi.org/10.1021/ac981425v

Savage, Nora y Mamadou S. Diallo. (2005). Nanomaterials and water purification: opportunities and challenges. J. Nanoparticle Research, 7: 331-342.

http://dx.doi.org/10.1007/s11051-005-7523-5

Senellart, Pascale; Glenn Solomon y Andrew White. (2017). High performance semiconductor quantum dots single photon source. Nature nanotecnology, 12: 1026-1039; http://dx.doi.org/10.1038/nnano.2017.218

Shao, Yuyan; Jun Wang, Hong Wu, Jun Liu, Ilhan A. Aksay, Yuehe Lin. (2010). Graphene based electrochemical sensors and biosensors: a review. Electroanalysis, 22: 1027-1036; http://dx.doi.org/10.1002/elan.200900571

Sharma, Bhavya; Renee R. Frontiera, Anne-Isabelle Henry, Emilie Ringe, Richard P. Van Duyne. (2012). SERS: Materials, applications, and the future. Materials Today, 15: 16-25. http://dx.doi.org/10.1016/S1369-7021(12)70017-2

Shi, Jinjun; Philip W. Kantoff, Richard Wooster y Omid C. Farokhzad. (2017). Cancer nanomedicine: progress, challenges and opportunities. Nature Reviews Cancer, 17: 20-37. http://dx.doi.org/10.1038/nrc.2016.108

Solis, D.; E. De la Rosa, O. Meza, L. A. Díaz-Torres, P. Salas, C. Angeles-Chávez. (2010). Role of $\mathrm{Yb3}+$ and Er3+ concentration on the tunability of green-yellow-red upconversion emission of codoped $\mathrm{ZrO} 2: \mathrm{Yb3}+-\mathrm{Er} 3+$ nanocrystals. J. Appl. Phys., 108: 023103. http://dx.doi.org/10.1063/1.3465325

Srivastava, Anup K.; Atul Dev, Surajit Karmakar. (2017). Nanosensors and nanobiosensors in food and agricultura. Enviromental Chem. Lett., 16: 161-182. http://dx.doi.org/10.1007/s10311-017-0674-7

Sun, Chunli; Xiaowen Ou, Yong Cheng, Tianyou Zhai, Bifeng Liu, Xiaoding Lou y Fan Xia. (2019). Coordination-induced structural changes of DNA-based optical and electrochemical sensors for metal ions detection. Dalton Trans., 48: 58795891. http://dx.doi.org/10.1039/C8DT04733B

US National Science and Technology Council, Committee on Technology, Interagen- 
cy Working Group on NanoScience, Engineering and Technology. (1999). Nanostructure science and technology, a worldwide study. Septiembre.

http://www.wtec.org/loyola/nano/

Wang, Feng y Xiaogang Liu. (2014). Multicolor tuning of lanthanide-doped nanoparticles by single wavelength excitation. Acc. Chem. Res., 47: 1378-1385. http://dx.doi.org/10.1021/ar5000067

Xie, Xueping; Jinfeng Liao, Xiaoru Shao, Qianshun Li y Yunfeng Lin. (2017). The effect of shape on cellular uptake of gold nanoparticles in the forms of stars, rods, and triangles. Scientific reports, 7: 3827.

http://dx.doi.org/10.1038/s41598-017-04229-z

Yang, Yixing; Ying Zheng, Weiran Cao, Alexandre Titov, Jake Hyvonen, Jesse R. Manders, Jiangeng Xue, Paul H. Holloway y Lei Qian. (2015). High efficiency light emitting devices based on quantum dots with tailored nanostructures. Nature Photonics, 9: 259-266. http://dx.doi.org/10.1038/nphoton.2015.36

Yin, Yadong y Dmitri Talapin. (2013). The chemistry of functional nanomaterials. Chem. Soc. rev., 42: 2484-2487; http://dx.doi.org/10.1039/C3CS90011H

Yuan, Jingli y Guilan Wang. (2006). Lanthanide-based luminescence probes and time-resolved luminescence bioassays. TrAC Trends in Analytical Chemistry, 25: 490-500. http://dx.doi.org/10.1016/j.trac.2005.11.013

Zhou, Qing; Yilin Hou, Li Zhang, Jianlin Wang, Youbei Qiao, Songyan Guo, Li Fan, Tiehong Yang, Lin Zhu y Hong Wu. (2017). Dual-pH sensitive charge-reversal nanocomplex for tumor-targeted drug delivery with enhanced anticancer activity. Theranostic, 7: 1806-1819. http://dx.doi.org/10.7150/thno.18607

Zukovskaja, O.; S. Agafilushkina, V. Sivakov, K. Weber, D. Cialla-May, L. Osminkina y J. Popp. (2019). Rapid detection of the bacterial biomarker pyocyanin in artificial sputum using a SERS-active silicon nanowire matrix covered by bimetallic noble metal nanoparticles. Talanta, 202: 171-177.

http://dx.doi.org/10.1016/j.talanta.2019.04.047

\section{Páginas Web}

http://www.nano.gov/timeline 